Portland State University

PDXScholar

$4-1-1990$

\title{
Spontaneous Coherent Pulsations in Standing-Wave Laser Oscillators: Simplified Models
}

Lee W. Casperson

Portland State University

Manuela Fe Tarroja

University of the Philippines Diliman

Follow this and additional works at: https://pdxscholar.library.pdx.edu/ece_fac

Part of the Electrical and Computer Engineering Commons

Let us know how access to this document benefits you.

\section{Citation Details}

Lee W. Casperson and Manuela Fe H. Tarroja (1991). Spontaneous coherent pulsations in standing-wave laser oscillators: simplified models, J. Opt. Soc. Am. B 8, 250-261.

This Article is brought to you for free and open access. It has been accepted for inclusion in Electrical and Computer Engineering Faculty Publications and Presentations by an authorized administrator of PDXScholar. Please contact us if we can make this document more accessible: pdxscholar@pdx.edu. 


\title{
Spontaneous coherent pulsations in standing-wave laser oscillators: simplified models
}

\author{
Lee W. Casperson and Manuela Fe H. Tarroja* \\ Department of Electrical Engineering, Portland State University, Portland, Oregon 97207
}

Received June 27, 1989; accepted April 2, 1990

\begin{abstract}
A series of simplifications and approximations is introduced into a recently described model for spontaneous pulsations in standing-wave laser oscillators. To the extent that these simplifications are valid they can lead to significant reductions in computation time and sometimes also to a better understanding of the relative importance of various physical effects. Of special interest is the number of spatial harmonics required to represent adequately the effects of longitudinal spatial hole burning. Other approximations investigated include neglect of spectral cross relaxation, neglect of multiple-energy-level equations, neglect of electric-field derivatives, and neglect of polarization derivatives. In addition, a detailed discussion is included that concerns the most efficient numerical implementation of the model.
\end{abstract}

\section{INTRODUCTION}

Under a variety of conditions xenon lasers operating at $3.51 \mu \mathrm{m}$ are observed to produce their output in the form of an infinite train of periodic or chaotic pulses, and similar behavior is observed with other laser types. In the case of xenon lasers the pulsations are understood to result from a fundamental instability in the semiclassical laser equations, ${ }^{1}$ and related effects may be important in other lasers. $^{2-5}$ Recently a more rigorous Maxwell-Schrödinger semiclassical model was developed to describe the behavior of standing-wave Doppler-broadened lasers. ${ }^{6,7}$ That model provides good agreement with experimental pulsation data and with Lamb-dip power curves.

While there are obvious advantages to having an instability model that is quantitatively accurate, there are also important reasons for studying simplified versions of such quantitative models. Unlike in the mathematical models for many other physical systems, the accuracy that one wishes to achieve controls the number of differential equations that constitute the model. For higher accuracy more equations are needed to represent more spatial harmonics or more velocity classes of atoms. Since computer requirements for integrating the semiclassical equations are quite substantial, it is important that the number of governing equations be kept to the minimum consistent with the desired accuracy. Further simplifications occur when one is interested only in the qualitative effects of certain terms in the model rather than quantitative accuracy. The purpose of this study is to start from the general standing-wave laser model that was recently developed $^{6}$ and, through a series of approximations, to reduce that model to the simplest form that still retains the basic features of the semiclassical standing-wave laser instability.

The general theoretical model for a standing-wave laser is briefly summarized in Section 2 together with some transformations that are useful for maximizing the efficiency of the brute-force numerical solutions. Section 3 includes a detailed derivation of the threshold parameter. A rigorous relationship between the pump rates and the threshold parameter is especially important, since this parameter is easily determined experimentally. Initial values of the laser variables are discussed in Section 4 together with a few representative solutions. The effects of varying the number of longitudinal spatial harmonics of the populations and polarizations are discussed in Section 5. For the xenon lasers of interest here, it is found that only the lowest harmonics are needed to obtain reasonable accuracy. Section 6 presents a sequence of further simplifications that include the neglect of spectral cross relaxation, neglect of multiple-energy-level equations, and neglect of electric-field derivatives. None of these approximations has a major qualitative effect on the instability. On the other hand, the rate-equation approximation discussed in Section 7 eliminates the instability.

\section{GENERAL MODEL}

The theoretical model on which this study is based was discussed in Ref. 6. In its normalized single-isotope gaslaser form, the model is represented by Eqs. (88)-(93) of Ref. 6, and these equations are

$$
\begin{aligned}
& \frac{\partial P_{r, 2 j+1}(V, t)}{\partial t}=-\gamma\left\{[1+(2 j+1) i V] P_{r, 2 j+1}(V, t)\right. \\
& +y P_{i, 2 j+1}(V, t)+i A_{i}(t) \\
& \left.\times\left[D_{2 j}(V, t)-D_{2 j+2}(V, t)\right]\right\}, \\
& \frac{\partial P_{i, 2 j+1}(V, t)}{\partial t}=-\gamma\left\{[1+(2 j+1) i V] P_{i, 2 j+1}(V, t)\right. \\
& -y P_{r, 2 j+1}(V, t)-i A_{r}(t) \\
& \left.\times\left[D_{2 j}(V, t)-D_{2 j+2}(V, t)\right]\right\},
\end{aligned}
$$




$$
\begin{aligned}
& \frac{\partial D_{2 j}(V, t)}{\partial t}= {\left[\lambda_{a}(V, t)-\lambda_{b}(V, t)\right] \delta_{j 0} } \\
&-\left[h_{1}+(2 j) i \gamma V\right] D_{2 j}(V, t)-h_{2} M_{2 j}(V, t) \\
&-i \gamma_{1}\left\{\left[A_{r}(t) P_{i, 2 j-1}(V, t)-A_{i}(t) P_{r, 2 j-1}(V, t)\right]\right. \\
&\left.\quad-\left[A_{r}(t) P_{i, 2 j+1}(V, t)-A_{i}(t) P_{r, 2 j+1}(V, t)\right]\right\} \\
&+\frac{\varepsilon \Gamma_{a}}{2 \pi^{1 / 2}} \exp \left(-\varepsilon^{2} V^{2}\right) \int_{-\infty}^{\infty}\left[M_{2 j}\left(V^{\prime}, t\right)\right. \\
&\left.+D_{2 j}\left(V^{\prime}, t\right)\right] \mathrm{d} V^{\prime} \\
&-\frac{\varepsilon \Gamma_{b}}{2 \pi^{1 / 2}} \exp \left(-\varepsilon^{2} V^{2}\right) \int_{-\infty}^{\infty}\left[M_{2 j}\left(V^{\prime}, t\right)\right. \\
&\left.-D_{2 j}\left(V^{\prime}, t\right)\right] \mathrm{d} V^{\prime}, \\
& \frac{\partial M_{2 j}(V, t)}{\partial t}=\left.\lambda_{a}(V, t)+\lambda_{b}(V, t)\right] \delta_{j 0} \\
&-\left[h_{3}+(2 j) i \gamma V\right] M_{2 j}(V, t)-h_{4} D_{2 j}(V, t) \\
&+\frac{\varepsilon \Gamma_{a}}{2 \pi^{1 / 2}} \exp \left(-\varepsilon^{2} V^{2}\right) \int_{-\infty}^{\infty}\left[M_{2 j}\left(V^{\prime}, t\right)\right. \\
&\left.+D_{2 j}\left(V^{\prime}, t\right)\right] \mathrm{d} V^{\prime} \\
&+\frac{\varepsilon \Gamma_{b}}{2 \pi^{1 / 2}} \exp \left(-\varepsilon^{2} V^{2}\right) \int_{-\infty}^{\infty}\left[M_{2 j}\left(V^{\prime}, t\right)\right. \\
&\left.-D_{2 j}\left(V^{\prime}, t\right)\right] \mathrm{d} V^{\prime}, \\
& \frac{\mathrm{d} A_{i}(t)}{\mathrm{d} t}=-\frac{1}{2 t_{c}}\left[A_{i}(t)-\delta\left(y-y_{0}\right) A_{r}(t)\right. \\
&\left.\quad+\int_{-\infty}^{\infty} P_{r, 1 i}(V, t) \mathrm{d} V\right] \\
& \mathrm{d} A_{r}(t) \\
&=-\frac{1}{2 t_{c}}\left[A_{r}(t)+\delta\left(y-y_{0}\right) A_{i}(t)\right. \\
&\left.-\int_{-\infty}^{\infty} P_{i, 1 i}(V, t) \mathrm{d} V\right] \\
& \\
& \mathrm{d} t(5) \\
&
\end{aligned}
$$

The dependent variables in Eqs. (1)-(6) include the $2 j+1$ spatial harmonic of the real part $P_{r, 2 j+1}(V, t)$ and the imaginary part $P_{i, 2 j+1}(V, t)$ of the polarization, the $2 j$ harmonic of the difference between the populations of the upper and lower laser levels $D_{2 j}(V, t)$ and of their sum $M_{2 j}(V, t)$, and the real part $A_{r}(t)$ and the imaginary part $A_{i}(t)$ of the electric field. The spatially independent pumping rates of the upper and lower levels are $\lambda_{a}(V, t)$ and $\lambda_{b}(V, t)$, respectively, and the basic decay rates include the total decay rate for the upper laser level $\gamma_{a}$, the total decay rate for the lower laser level $\gamma_{b}$, the rate of direct decay from the upper level to the lower level $\gamma_{a b}$, the polarization decay rate $\gamma$, and the electric-field decay rate $\left(2 t_{c}\right)^{-1}$, where $t_{c}$ is the cavity lifetime.

Equations (1)-(6) also include the possibility of strong velocity-changing collisions in which the final velocities are distributed randomly across the Doppler profile. Thus the coefficient $\Gamma_{a}$ represents the total rate at which atoms in level $a$ undergo strong velocity-changing collisions, and $\Gamma_{b}$ is the corresponding total rate for level $b$. The velocity integrals in Eqs. (3) and (4) represent the collision-caused addition of atoms to each velocity class, and the collision-caused loss from each class is accounted for by replacing the total spontaneous emission decay rates $\gamma_{a}$ and $\gamma_{b}$ with the new values $\gamma_{a}{ }^{\prime}=\gamma_{a}+\Gamma_{a}$ and $\gamma_{b}{ }^{\prime}=\gamma_{b}+\Gamma_{b}$. These decay rates are represented in the model by the hybrid decay rates

$$
\begin{aligned}
& h_{1}=\left(\gamma_{a}^{\prime}+\gamma_{a b}+\gamma_{b}{ }^{\prime}\right) / 2, \\
& h_{2}=\left(\gamma_{a}^{\prime}+\gamma_{a b}-\gamma_{b}{ }^{\prime}\right) / 2, \\
& h_{3}=\left(\gamma_{a}^{\prime}-\gamma_{a b}+\gamma_{b}{ }^{\prime}\right) / 2, \\
& h_{4}=\left(\gamma_{a}^{\prime}-\gamma_{a b}-\gamma_{b}{ }^{\prime}\right) / 2, \\
& \gamma_{1}=2 \gamma_{a}^{\prime} \gamma_{b}^{\prime} /\left(\gamma_{a}^{\prime}-\gamma_{a b}+\gamma_{b}{ }^{\prime}\right) .
\end{aligned}
$$

The parameter $y=\left(\omega-\omega_{0}\right) / \gamma$ in Eqs. (1) and (6) represents the normalized difference between a selected lasing reference frequency $\omega$ and the line-center frequency $\omega_{0}$, and $y_{0}$ represents the corresponding parameter value when dispersion effects are neglected. The normalized axial component of velocity is $V$. The natural damping ratio $\varepsilon=\left(\Delta \nu_{h} / \Delta \nu_{d}\right)(\ln 2)^{1 / 2}$ measures the relative magnitudes of the homogeneous and inhomogeneous linewidths, and the parameter $\delta=2 \gamma t_{c}$ measures the ratio of the polarization and electric-field decay rates. Equations (1)(4) follow from the semiclassical equations that govern the four elements of the density matrix for the two active levels of the laser transition in the presence of a standingwave electric field. Equations (5) and (6) follow from Maxwell's equations for the field amplitude in the presence of an atomic or molecular laser medium. ${ }^{6}$

In Ref. 6, Eqs. (1)-(6) were solved to obtain the time characteristics of the spontaneous coherent pulsations that often occur in high-gain xenon lasers, and the corresponding frequency characteristics were discussed in Ref. 7. Various simplifications of these equations are introduced here to reduce programming complexity, speed up computation rates, or provide greater insight into underlying physical mechanisms. As with our earlier ring laser model, it is helpful initially to separate the populations into a broad Gaussian part that represents the pump distribution and a narrow part that reflects the localized distortion caused by the laser fields. Thus the spatial harmonics of the population difference and sum are written as $^{8}$

$$
\begin{aligned}
& D_{2 j}(V, t)=\frac{\varepsilon E_{2 j}(t)}{\pi^{1 / 2}} \exp \left(-\varepsilon^{2} V^{2}\right)+D_{2 j}{ }^{\prime}(V, t), \\
& M_{2 j}(V, t)=\frac{\varepsilon F_{2 j}(t)}{\pi^{1 / 2}} \exp \left(-\varepsilon^{2} V^{2}\right)+M_{2 j}{ }^{\prime}(V, t),
\end{aligned}
$$

and the pumping rates are written as

$$
\begin{aligned}
& \lambda_{a}(V, t)=\frac{\varepsilon L_{a}(t)}{\pi^{1 / 2}} \exp \left(-\varepsilon^{2} V^{2}\right), \\
& \lambda_{b}(V, t)=\frac{\varepsilon L_{b}(t)}{\pi^{1 / 2}} \exp \left(-\varepsilon^{2} V^{2}\right),
\end{aligned}
$$

where $L_{a}(t)$ and $L_{b}(t)$ represent the total rates of pumping into the upper and lower laser levels, respectively. When these definitions are substituted into Eqs. (3) and (4), one can arbitrarily define $E_{2 j}(t)$ and $F_{2 j}(t)$ to obtain the separation 


$$
\begin{aligned}
\frac{\partial E_{2 j}(t)}{\partial t}= & {\left[L_{a}(t)-L_{b}(t)\right] \delta_{j 0}-h_{1} E_{2 j}(t)-h_{2} F_{2 j}(t) } \\
& +\frac{\Gamma_{a}}{2}\left\{F_{2 j}(t)+E_{2 j}(t)+\int_{-\infty}^{\infty}\left[M_{2 j}{ }^{\prime}\left(V^{\prime}, t\right)\right.\right. \\
& \left.\left.+D_{2 j}{ }^{\prime}\left(V^{\prime}, t\right)\right] \mathrm{d} V^{\prime}\right\}-\frac{\Gamma_{b}}{2}\left\{F_{2 j}(t)-E_{2 j}(t)\right. \\
& \left.+\int_{-\infty}^{\infty}\left[M_{2 j}\left(V^{\prime}, t\right)-D_{2 j}\left(V^{\prime}, t\right)\right] \mathrm{d} V^{\prime}\right\},
\end{aligned}
$$$$
\frac{\partial F_{2 j}(t)}{\partial t}=\left[L_{a}(t)+L_{b}(t)\right] \delta_{j 0}-h_{3} F_{2 j}(t)-h_{4} E_{2 j}(t)
$$$$
+\frac{\Gamma_{a}}{2}\left\{F_{2 j}(t)+E_{2 j}(t)+\int_{-\infty}^{\infty}\left[M_{2 j} j^{\prime}\left(V^{\prime}, t\right)\right.\right.
$$$$
\left.\left.+D_{2 j^{\prime}}\left(V^{\prime}, t\right)\right] \mathrm{d} V^{\prime}\right\}+\frac{\Gamma_{b}}{2}\left\{F_{2 j}(t)-E_{2 j}(t)\right.
$$$$
\left.+\int_{-\infty}^{\infty}\left[M_{2 j^{\prime}}\left(V^{\prime}, t\right)-D_{2 j^{\prime}}\left(V^{\prime}, t\right)\right] \mathrm{d} V^{\prime}\right\},
$$

$\frac{2 D_{2 j}{ }^{\prime}(V, t)}{\partial t}=-\left[h_{1}+(2 j) i \gamma V\right] D_{2 j}{ }^{\prime}(\mathrm{V}, t)-h_{2} M_{2 j}{ }^{\prime}(V, t)$

$$
\begin{aligned}
& -\frac{(2 j) i \gamma V E_{2 j}(t) \varepsilon}{\pi^{1 / 2}} \exp \left(-\varepsilon^{2} V^{2}\right) \\
& -i \gamma_{1}\left\{\left[A_{r}(t) P_{i, 2 j-1}(V, t)-A_{i}(t) P_{r, 2 j-1}(V, t)\right]\right. \\
& \left.-\left[A_{r}(t) P_{i, 2 j+1}(V, t)-A_{i}(t) P_{r, 2 j+1}(V, t)\right]\right\},
\end{aligned}
$$

$\frac{\partial M_{2 j}{ }^{\prime}(V, t)}{\partial t}=-\left[h_{3}+(2 j) i \gamma V\right] M_{2 j}{ }^{\prime}(V, t)-h_{4} D_{2 j}{ }^{\prime}(V, t)$

$$
-\frac{(2 j) i \gamma V F_{2 j}(t) \varepsilon}{\pi^{1 / 2}} \exp \left(-\varepsilon^{2} V^{2}\right) .
$$

With the introduction of new decay rates, Eq. (16) and (17) can be further reduced to

$$
\begin{aligned}
\frac{\mathrm{d} E_{2 j}(t)}{\mathrm{d} t}= & {\left[L_{a}(t)-L_{b}(t)\right] \delta_{j 0}-h_{5} E_{2 j}(t)-h_{6} F_{2 j}(t) } \\
& +\frac{\Gamma_{a}}{2} \int_{-\infty}^{\infty}\left[M_{2 j^{\prime}}\left(V^{\prime}, t\right)+D_{2 j}{ }^{\prime}\left(V^{\prime}, t\right)\right] \mathrm{d} V^{\prime} \\
& -\frac{\Gamma_{b}}{2} \int_{-\infty}^{\infty}\left[M_{2 j}^{\prime}\left(V^{\prime}, t\right)-D_{2 j}{ }^{\prime}\left(V^{\prime}, t\right)\right] \mathrm{d} V^{\prime},
\end{aligned}
$$

$$
\begin{aligned}
\frac{\mathrm{d} F_{2 j}(t)}{\mathrm{d} t}= & {\left[L_{a}(t)+L_{b}(t)\right] \delta_{j 0}-h_{7} F_{2 j}(t)-h_{8} E_{2 j}(t) } \\
& +\frac{\Gamma_{a}}{2} \int_{-\infty}^{\infty}\left[M_{2 j}{ }^{\prime}\left(V^{\prime}, t\right)+D_{2 j}{ }^{\prime}\left(V^{\prime}, t\right)\right] \mathrm{d} V^{\prime} \\
& +\frac{\Gamma_{b}}{2} \int_{-\infty}^{\infty}\left[M_{2 j}{ }^{\prime}\left(V^{\prime}, t\right)-D_{2 j}{ }^{\prime}\left(V^{\prime}, t\right)\right] \mathrm{d} V^{\prime}
\end{aligned}
$$

where the new rates are defined by

$$
\begin{aligned}
& h_{5}=h_{1}-\Gamma_{a} / 2-\Gamma_{b} / 2=\left(\gamma_{a}+\gamma_{a b}+\gamma_{b}\right) / 2, \\
& h_{6}=h_{2}-\Gamma_{a} / 2+\Gamma_{b} / 2=\left(\gamma_{a}+\gamma_{a b}-\gamma_{b}\right) / 2, \\
& h_{7}=h_{3}-\Gamma_{a} / 2-\Gamma_{b} / 2=\left(\gamma_{a}-\gamma_{a b}+\gamma_{b}\right) / 2, \\
& h_{8}=h_{4}-\Gamma_{a} / 2+\Gamma_{b} / 2=\left(\gamma_{a}-\gamma_{a b}-\gamma_{b}\right) / 2 .
\end{aligned}
$$

With these transformations the velocity integrations need extend only across the saturated range of velocities, and all our numerical solutions involving the cross-relaxation integrals incorporate this separation.

It has also proved to be convenient to introduce a new numbering of the spatial harmonics that takes advantage of the fact that only odd polarization harmonics are important. Thus the same subscript $k$ can replace $2 j+1$ in the polarization terms and $2 j$ in the population terms; the governing equations, Eqs. (1), (2), (5), (6), and (18)-(21), reduce to

$$
\begin{aligned}
\frac{\partial P_{r, k}(V, t)}{\partial t}=-\gamma\{ & {[1+(2 k-1) i V] P_{r, k}(V, t)+y P_{i, k}(V, t) } \\
& +i A_{i}(t)\left[\varepsilon E_{k}(t) \frac{\exp \left(-\varepsilon^{2} V^{2}\right)}{\pi^{1 / 2}}\right. \\
& -\varepsilon E_{k+1}(t) \frac{\exp \left(-\varepsilon^{2} V^{2}\right)}{\pi^{1 / 2}} \\
& \left.\left.+D_{k}^{\prime}(V, t)-D_{k+1}^{\prime}(V, t)\right]\right\}, \\
\frac{\partial P_{i, k}(V, t)}{\partial t}=-\gamma\left\{[1+(2 k-1) i V] P_{i, k}(V, t)-y P_{r, k}(V, t)\right. & \\
& -i A_{r}(t)\left[\varepsilon E_{k}(t) \frac{\exp \left(-\varepsilon^{2} V^{2}\right)}{\pi^{1 / 2}}\right. \\
& -\varepsilon E_{k+1}(t) \frac{\exp \left(-\varepsilon^{2} \dot{V}^{2}\right)}{\pi^{1 / 2}} \\
& \left.\left.+D_{k}^{\prime}(V, t)-D_{k+1}{ }^{\prime}(V, t)\right]\right\},
\end{aligned}
$$

$$
\begin{aligned}
\frac{\partial D_{k}{ }^{\prime}(V, t)}{\partial t}= & -\left[h_{1}+(2 k-2) i \gamma V\right] D_{k}{ }^{\prime}(V, t)-h_{2} M_{k}^{\prime}(V, t) \\
& -(2 k-2) i \gamma V E_{k}(t) \frac{\varepsilon \exp \left(-\varepsilon^{2} V^{2}\right)}{\pi^{1 / 2}} \\
& -i \gamma_{1}\left[A_{r}(t) P_{i, k-1}(V, t)-A_{i}(t) P_{r, k-1}(V, t)\right. \\
& \left.-A_{r}(t) P_{i, k}(V, t)+A_{i}(t) P_{r, k}(V, t)\right]
\end{aligned}
$$

$$
\begin{aligned}
\frac{\partial M_{k}^{\prime}(V, t)}{\partial t}= & -\left[h_{3}+(2 k-2) i \gamma V\right] M_{k}{ }^{\prime}(V, t)-h_{4} D_{k^{\prime}}(V, t) \\
& -(2 k-2) i \gamma V F_{k}(t) \frac{\varepsilon \exp \left(-\varepsilon^{2} V^{2}\right)}{\pi^{1 / 2}},
\end{aligned}
$$

$$
\begin{aligned}
\frac{\mathrm{d} E_{k}(t)}{\mathrm{d} t}= & {\left[L_{a}(t)-L_{b}(t)\right] \delta_{k 1}-h_{5} E_{k}(t)-h_{6} F_{k}(t) } \\
& +\frac{\Gamma_{a}}{2} \int_{-\infty}^{\infty}\left[M_{k}{ }^{\prime}\left(V^{\prime}, t\right)+D_{k}{ }^{\prime}\left(V^{\prime}, t\right)\right] \mathrm{d} V^{\prime} \\
& -\frac{\Gamma_{b}}{2} \int_{-\infty}^{\infty}\left[M_{k}{ }^{\prime}\left(V^{\prime}, t\right)-D_{k}{ }^{\prime}\left(V^{\prime}, t\right)\right] \mathrm{d} V^{\prime},
\end{aligned}
$$

$$
\begin{aligned}
\frac{\mathrm{d} F_{k}(t)}{\mathrm{d} t}= & {\left[L_{a}(t)+L_{b}(t)\right] \delta_{k 1}-h_{7} F_{k}(t)-h_{8} E_{k}(t) } \\
& +\frac{\Gamma_{a}}{2} \int_{-\infty}^{\infty}\left[M_{k}{ }^{\prime}\left(V^{\prime}, t\right)+D_{k}{ }^{\prime}\left(V^{\prime}, t\right)\right] \mathrm{d} V^{\prime} \\
& +\frac{\Gamma_{b}}{2} \int_{-\infty}^{\infty}\left[M_{k}{ }^{\prime}\left(V^{\prime}, t\right)-D_{k}\left(V^{\prime}, t\right)\right] \mathrm{d} V^{\prime},
\end{aligned}
$$




$$
\begin{aligned}
& \frac{\mathrm{d} A_{r}(t)}{\mathrm{d} t}=-\frac{1}{2 t_{c}}[ A_{r}(t)+\delta\left(y-y_{0}\right) A_{i}(t) \\
&\left.-\int_{-\infty}^{\infty} P_{i, 1 i}(V, t) \mathrm{d} V\right], \\
& \frac{\mathrm{d} A_{i}(t)}{\mathrm{d} t}=-\frac{1}{2 t_{c}}\left[A_{i}(t)-\delta\left(y-y_{0}\right) A_{r}(t)\right. \\
&\left.+\int_{-\infty}^{\infty} P_{r, 1 i}(V, t) \mathrm{d} V\right] .
\end{aligned}
$$

It is not actually necessary to carry out computations for negative values of the integer $k$. Equations (26)-(33) are valid as written for values of $k$ that are larger than unity, and values of $k$ that are smaller than unity can be eliminated by means of the following relationships ${ }^{6}$ :

$$
\begin{aligned}
P_{r, j}(V, t) & =P_{r,-j}{ }^{*}(V, t), \\
P_{i, j}(V, t) & =P_{i,-j} *(V, t), \\
D_{j}(V, t) & =D_{-j}^{*}(V, t), \\
M_{j}(V, t) & =M_{-j}^{*}(V, t) .
\end{aligned}
$$

However, for $k=1 \mathrm{Eq}$. (28) couples to a more negative value of $k$. In this case Eq. (28) can be replaced by its equivalent form,

$$
\begin{aligned}
\frac{\partial D_{1}^{\prime}(V, t)}{\partial t}= & -h_{1} D_{1}^{\prime}(V, t)-h_{2} M_{1}^{\prime}(V, t) \\
& -2 \gamma_{1}\left[A_{r}(t) P_{i, 1 i}(V, t)-A_{i}(t) P_{r, 1 i}(V, t)\right] .
\end{aligned}
$$

With this modification, Eqs. (26)-(33) form a complete set that uses $k$ values of unity and larger. It is in this form that the equations have been programmed and numerically solved for our studies of spontaneous pulsations in standing-wave xenon lasers.

\section{THRESHOLD PARAMETER}

In order to obtain useful solutions to the equations described above, it is necessary to establish simple and realistic values for the various quantities involved. It is especially helpful if these quantities can be expressed in terms of a threshold parameter that relates the laser pump rates to their values when the laser is exactly at the lasing threshold. This threshold parameter provides a simple and direct link between laboratory experiments and computations.

Several simplifications of Eqs. (1)-(6) occur for operation at the lasing threshold. At threshold the fields and polarizations are infinitesimal, so that the saturation terms in Eq. (3) may be deleted, leaving

$$
\begin{aligned}
& \frac{\partial D_{2 j}(V, t)}{\partial t}=\left[\lambda_{a}(V)-\lambda_{b}(V)\right] \delta_{j 0} \\
& \quad-\left[h_{1}+(2 j) i \gamma V\right] D_{2 j}(V, t)-h_{2} M_{2 j}(V, t) \\
& \quad+\frac{\varepsilon \Gamma_{a}}{2 \pi^{1 / 2}} \exp \left(-\varepsilon^{2} V^{2}\right) \int_{-\infty}^{\infty}\left[M_{2 j}\left(V^{\prime}, t\right)+D_{2 j}\left(V^{\prime}, t\right)\right] \mathrm{d} V^{\prime} \\
& \quad-\frac{\varepsilon \Gamma_{b}}{2 \pi^{1 / 2}} \exp \left(-\varepsilon^{2} V^{2}\right) \int_{-\infty}^{\infty}\left[M_{2 j}\left(V^{\prime}, t\right)-D_{2 j}\left(V^{\prime}, t\right)\right] \mathrm{d} V^{\prime},
\end{aligned}
$$

where it is assumed that the pump rates are independent of time. In the absence of saturation the rate at which atoms are removed from any velocity class by strong velocity-changing collisions is the same as the rate at which they are added. Therefore, for purposes of the threshold calculation, the Gaussian cross-relaxation integrals in Eqs. (4) and (39) may be performed, and the results may be combined with the other population difference and sum terms. This results in the simpler equations

$$
\begin{aligned}
\frac{\partial D_{2 j}(V, t)}{\partial t}= & {\left[\lambda_{a}(V)-\lambda_{b}(V)\right] \delta_{j 0} } \\
& -\left[h_{5}+(2 j) i \gamma V\right] D_{2 j}(V, t)-h_{6} M_{2 j}(V, t), \\
\frac{\partial M_{2 j}(V, t)}{\partial t}= & {\left[\lambda_{a}(V)+\lambda_{b}(V)\right] \delta_{j 0} } \\
& -\left[h_{7}+(2 j) i \gamma V\right] M_{2 j}(V, t)-h_{8} D_{2 j}(V, t),
\end{aligned}
$$

where the population decay rates are defined in Eqs. (22)(25). It is now apparent from Eqs. (40) and (41) that at threshold only the $j=0$ spatial harmonic will be excited. Also, the threshold condition can be obtained from a steady-state calculation, and from Eqs. (1), (2), (5), (6), (40), and (41) the set of governing equations is simply

$$
\begin{aligned}
& 0=(1+i V) P_{r, 1}(V)+y P_{i, 1}(V)+i A_{i} D_{0}(V), \\
& 0=(1+i V) P_{i, 1}(V)-y P_{r, 1}(V)-i A_{r} D_{0}(V), \\
& 0=\lambda_{a}(V)-\lambda_{b}(V)-h_{5} D_{0}(V)-h_{6} M_{0}(V), \\
& 0=\lambda_{a}(V)+\lambda_{b}(V)-h_{7} M_{0}(V)-h_{8} D_{0}(V), \\
& 0=A_{r}+\delta\left(y-y_{0}\right) A_{i}-\int_{-\infty}^{\infty} P_{i, 1 i}(V) \mathrm{d} V, \\
& 0=A_{i}-\delta\left(y-y_{0}\right) A_{r}+\int_{-\infty}^{\infty} P_{r, 1 i}(V) \mathrm{d} V .
\end{aligned}
$$

Equations (42)-(47) can be solved by means of straightforward substitutions. Thus Eqs. (42) and (43) can be combined to obtain

$$
\begin{aligned}
P_{r, 1}(V)= & -\frac{i}{1+i V}\left(\frac{y}{1+i V} A_{r}+A_{i}\right) \\
& \times D_{0}(V) \frac{1}{1+[y /(1+i V)]^{2}} \\
= & -i \alpha_{0}(V, y)\left[\beta_{0}(V, y) A_{r}+A_{i}\right] D_{0}(V), \\
P_{i, 1}(V)= & -\frac{i}{1+i V}\left(\frac{y}{1+i V} A_{i}-A_{r}\right) \\
& \times D_{0}(V) \frac{1}{1+[y /(1+i V)]^{2}} \\
= & -i \alpha_{0}(V, y)\left[\beta_{0}(V, y) A_{i}-A_{r}\right] D_{0}(V),
\end{aligned}
$$

where we use the standing-wave laser notation ${ }^{9}$

$$
\alpha_{j}(V, y)=\frac{1 / 2}{1+(2 j+1) i V+i y}+\frac{1 / 2}{1+(2 j+1) i V-i y},
$$




$$
\beta_{j}(V, y)=\frac{y}{1+(2 j+1) i V} .
$$

Also, Eqs. (44) and (45) may be combined to obtain

$$
\begin{aligned}
D_{0}(V) & =\frac{\left(h_{7}-h_{6}\right) \lambda_{a}(V)-\left(h_{7}+h_{6}\right) \lambda_{b}(V)}{h_{5} h_{7}-h_{6} h_{8}} \\
& =\left(1-\frac{\gamma_{a b}}{\gamma_{b}}\right) \frac{\lambda_{a}(V)}{\gamma_{a}}-\frac{\lambda_{b}(V)}{\gamma_{b}},
\end{aligned}
$$

where the decay-rate relationships from Eqs. (22)-(25) are employed.

When Eqs. (48) and (49) are substituted into Eqs. (46) and (47), one obtains

$$
\begin{aligned}
0= & A_{r}+\delta\left(y-y_{0}\right) A_{i} \\
& +A_{i} \int_{-\infty}^{\infty} \operatorname{Re}\left[\alpha_{0}(V, y) \beta_{0}(V, y)\right] D_{0}(V) \mathrm{d} V \\
& -A_{r} \int_{-\infty}^{\infty} \operatorname{Re}\left[\alpha_{0}(V, y)\right] D_{0}(V) \mathrm{d} V, \\
0= & A_{i}-\delta\left(y-y_{0}\right) A_{r} \\
& -A_{r} \int_{-\infty}^{\infty} \operatorname{Re}\left[\alpha_{0}(V, y) \beta_{0}(V, y)\right] D_{0}(V) \mathrm{d} V \\
& -A_{i} \int_{-\infty}^{\infty} \operatorname{Re}\left[\alpha_{0}(V, y)\right] D_{0}(V) \mathrm{d} V .
\end{aligned}
$$

This is a homogeneous set of equations for the real and imaginary parts of the complex field amplitude. By setting the determinant of the coefficients of the field amplitudes to zero, one finds that nontrivial solutions are obtained only when subject to the constraints

$$
\begin{gathered}
1=\int_{-\infty}^{\infty} \operatorname{Re}\left[\alpha_{0}(V, y)\right] D_{0}(V) \mathrm{d} V, \\
\delta\left(y-y_{0}\right)=\int_{-\infty}^{\infty} \operatorname{Re}\left[\alpha_{0}(V, y) \beta_{0}(V, y)\right] D_{0}(V) \mathrm{d} V .
\end{gathered}
$$

Equations (55) and (56) define the conditions under which a laser operating at the normalized frequency $y$ will have exactly zero intensity. The threshold pumping condition is defined here by the zero-intensity constraint for a laser tuned to line center. At line center one finds $y_{0}=0, y=0$, and from Eq. (51) $\beta_{0}(V, y)=0$. Thus Eq. (56) is automatically satisfied. Using Eqs. (50) and (52), we find that Eq. (55) becomes

$$
1=\int_{-\infty}^{\infty} \operatorname{Re}\left(\frac{1}{1+i V}\right)\left[\left(1-\frac{\gamma_{a b}}{\gamma_{b}}\right) \frac{\lambda_{a}(V)}{\gamma_{a}}-\frac{\lambda_{b}(V)}{\gamma_{b}}\right] \mathrm{d} V .
$$

From Eqs. (14) and (15), the pump rates are Gaussian functions, and Eq. (57) reduces further to

$$
\begin{aligned}
1 & =\frac{\varepsilon}{\pi^{1 / 2}}\left[\left(1-\frac{\gamma_{a b}}{\gamma_{b}}\right) \frac{L_{a}}{\gamma_{a}}-\frac{L_{b}}{\gamma_{b}}\right] \int_{-\infty}^{\infty} \frac{\exp \left(-\varepsilon^{2} V^{2}\right)}{1+V^{2}} \mathrm{~d} V \\
& =\frac{\varepsilon}{\pi^{1 / 2}} L_{a}\left[\left(1-\frac{\gamma_{a b}}{\gamma_{b}}\right) \frac{1}{\gamma_{a}}-\frac{q}{\gamma_{b}}\right] \int_{-\infty}^{\infty} \frac{\exp \left(-\varepsilon^{2} V^{2}\right)}{1+V^{2}} \mathrm{~d} V,
\end{aligned}
$$

where the proportionality constant $q$ between the pump rates $L_{a}$ and $L_{b}$ has now been introduced.
Equation (58) can be understood as a definition of the threshold pump $L_{a \text { th }}$. The threshold parameter is usually defined as the ratio of the actual pump rate $L_{a}$ to the threshold pump rate $L_{a \text { th }}$. With Eq. (58) the threshold parameter $r$ is given by the formula

$$
\begin{aligned}
L_{a} & =r L_{a \text { th }} \\
& =r \frac{\pi^{1 / 2}}{\varepsilon}\left\{\left[\left(1-\frac{\gamma_{a b}}{\gamma_{b}}\right) \frac{1}{\gamma_{a}}-\frac{q}{\gamma_{b}}\right] \int_{-\infty}^{\infty} \frac{\exp \left(-\varepsilon^{2} V^{2}\right)}{1+V^{2}} \mathrm{~d} V\right\}^{-1} .
\end{aligned}
$$

Our numerical results are generally expressed in terms of the threshold parameter $r$, and Eq. (59) is used at the outset to express the pump rate in terms of $r$.

\section{INITIAL CONDITIONS}

Before computing the time-dependent solutions of Eqs. (26)-(33), it is necessary to choose initial values for certain dependent variables. A useful approach is based on the idea of loss switching ( $Q$ switching). This approach is widely used experimentally to obtain short pulses, and it also encourages the initiation of instabilities. The material is assumed to be pumped to some initial distribution of population, which corresponds to a particular value of the threshold parameter, while the field is held at zero. Then a small field is injected to begin the computations. Thus at the outset the only nonzero dependent variables are the coefficients of the Gaussian population distribution in Eqs. (12) and (13).

With saturation effects set to zero, the steady-state forms of Eqs. (30) and (31) are

$$
\begin{aligned}
& 0=L_{a}-L_{b}-\frac{\gamma_{a}+\gamma_{a b}+\gamma_{b}}{2} E_{1}-\frac{\gamma_{a}+\gamma_{a b}-\gamma_{b}}{2} F_{1}, \\
& 0=L_{a}+L_{b}-\frac{\gamma_{a}-\gamma_{a b}-\gamma_{b}}{2} E_{1}-\frac{\gamma_{a}-\dot{\gamma}_{a b}+\gamma_{b}}{2} F_{1} .
\end{aligned}
$$

These equations may be solved for $E_{1}$ and $F_{1}$, and the results are

$$
\begin{aligned}
& E_{1}=\left(1-\frac{\gamma_{a b}}{\gamma_{b}}\right) \frac{L_{a}}{\gamma_{a}}-\frac{L_{b}}{\gamma_{b}}, \\
& F_{1}=\left(1+\frac{\gamma_{a b}}{\gamma_{b}}\right) \frac{L_{a}}{\gamma_{a}}+\frac{L_{b}}{\gamma_{b}} .
\end{aligned}
$$

These are the population coefficients for a prepumped laser, and they are used at the start of our computations. Typically the other dependent variables in Eqs. (26)-(33) are initially set to zero except for a small initial value of the electric-field amplitude.

The model described here was developed especially for low-pressure standing-wave xenon lasers, although its form is general enough to provide a reasonable description of many other standing-wave laser systems. The coefficient values for our xenon laser calculations were summarized in Ref. 6, and these values include $\tau_{a}=\gamma_{a}^{-1}=1200 \mathrm{nsec}$, $\tau_{b}=\gamma_{b}{ }^{-1}=35 \mathrm{nsec}, \gamma_{a b}=\gamma_{a}, \gamma=16.3 \times 10^{6} \mathrm{sec}^{-1}, \Gamma_{a}=$ $\Gamma_{b}=1 \times 10^{6} \mathrm{sec}^{-1}, \varepsilon=0.039$, and $t_{c}=1.0 \mathrm{nsec}$.

Before beginning a discussion of the effects of various approximations, it is perhaps worthwhile again to indicate 

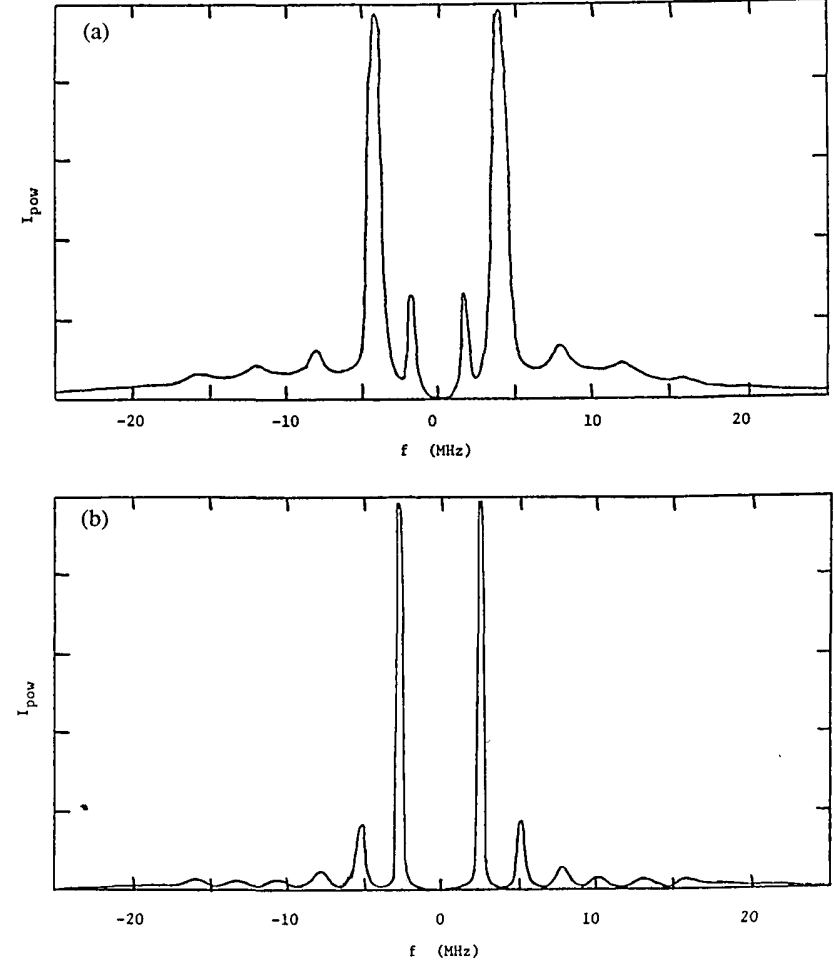

Fig. 1. Experimental power spectra of the output from a lowpressure xenon laser at discharge currents of (a) $50 \mathrm{~mA}(r \sim 2.1)$ and (b) $40 \mathrm{~mA}(r-1.7)$ (from Ref. 7).

briefly the agreement between experimental results and predictions of the general model. Since our emphasis has usually been on time-domain results, we present here experimental and theoretical power spectra for a lowpressure xenon laser. Figure 1(a) shows a typical experimental intensity-power spectrum for a laser operated with a threshold parameter of $r=2.1$, and the corresponding theoretical plot is given in Fig. 2(a). The experimental data, the calculation procedure, and the normalization are discussed further in Ref. 7. The frequency detuning was not measured in our experiments and is the only adjustable parameter in obtaining the corresponding theoretical spectrum. For the example in Fig. 2(a), the detuning $y_{0}=2.0$ was used. The small peak at $\sim 2 \mathrm{MHz}$ in the experimental and theoretical spectra is an indication that for these conditions the output pulsation bursts are alternating in form. Figure 1(b) shows an experimental power spectrum for a laser operated with a threshold parameter of $r=1.7$, and the corresponding theoretical spectrum with $y_{0}=15.0$ is shown in Fig. 2(b). The xenon laser is well characterized, and over a wide range of operating conditions no significant discrepancies between theory and experiment were observed in either the time or the frequency domain.

\section{SIMPLIFICATION OF SPATIAL HARMONICS}

The principal complication of rigorous standing-wave laser models in comparison with ring laser models is the necessary inclusion in the standing-wave models of multiple spatial harmonics of the atomic populations and polarizations. These harmonics are needed to account for $z$-dependent interaction effects such as longitudinal spatial hole burning. The inclusion of many spatial harmonics leads to a dramatic increase in computation time, in part because it increases the number of equations represented by Eqs. (26)-(33). More significantly, however, the increasing complexity of the model necessitates much smaller time steps in the integration algorithm if one is to obtain satisfactory convergence of the numerical solutions. Accordingly, it is important to be able to estimate the number of harmonics that are required for an application of the model.

One would expect that more spatial harmonics would be needed for larger values of the threshold parameter. Farther above threshold the stronger spatial hole burning makes the atomic populations increasingly nonuniform. Close to threshold, on the other hand, little saturation occurs, and the populations are almost constant in space. Figure 3 shows theoretical plots of the normalized intensity $I=A^{2}$ for a standing-wave laser that is tuned to line center $\left(y_{0}=0\right)$ and is operated far above threshold $(r=2.0)$. In Fig. 3(a) the numerical model has been truncated to a maximum number of spatial harmonics corresponding to $k_{\max }=8$. The corresponding results for the same initial conditions and decreasing values of $k_{\max }$ are shown in Figs. 3(b)-3(e). It is apparent from these figures that essentially exact results are obtained with $k_{\max }=6$ [Fig. 3(b)] out to a time of greater than $3.2 \mu \mathrm{sec}$ ( 9 pulsation bursts). Similar agreement is obtained with $k_{\max }=4$ [Fig. 3 (c)] out to a time of $\sim 2.7 \mu$ sec ( 8 bursts). Agreement with $k_{\max }=2$ [Fig. 3(d)] lasts until $\sim 1.3 \mu \mathrm{sec}$ (4 bursts). Agreement with $k_{\max }=1$ [Fig. 3(e)] lasts until $\sim 0.5 \mu \mathrm{sec}$ ( 1 burst). Thus for rigorous long-term accuracy it would seem that many spatial harmonics would be required. However, such extreme numerical accuracy is probably not needed in view of other approximations that are intrinsic in our best model and inevitable uncertain-

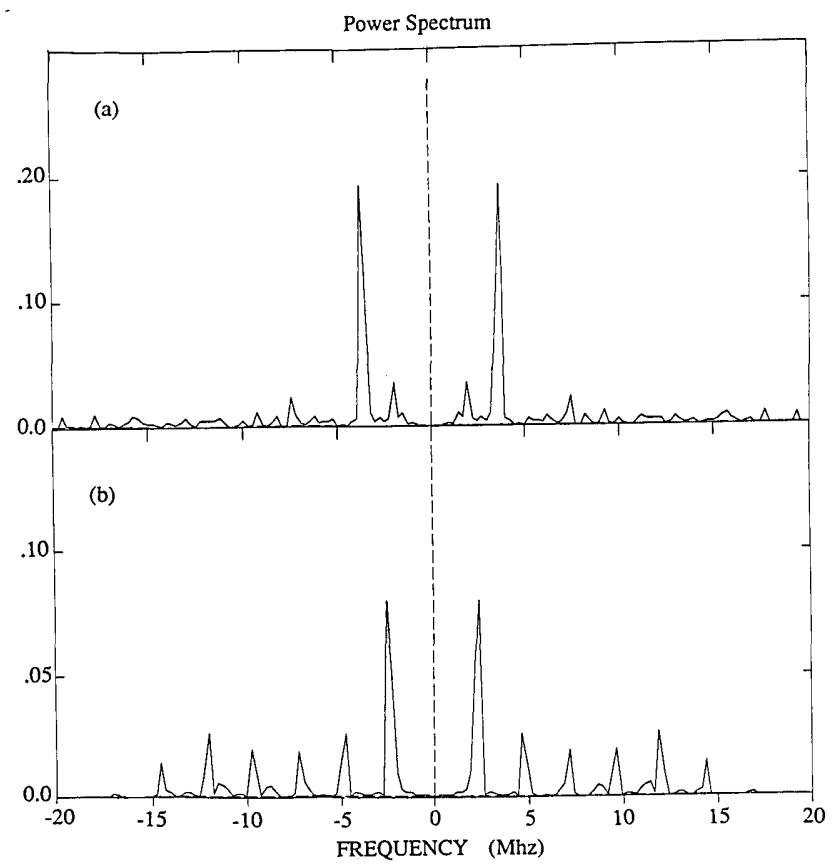

Fig. 2. Theoretical power spectra corresponding to threshold parameters and detunings of (a) $r=2.1, y_{0}=2.0$ and (b) $r=1.7$, $y_{0}=15.0$. 


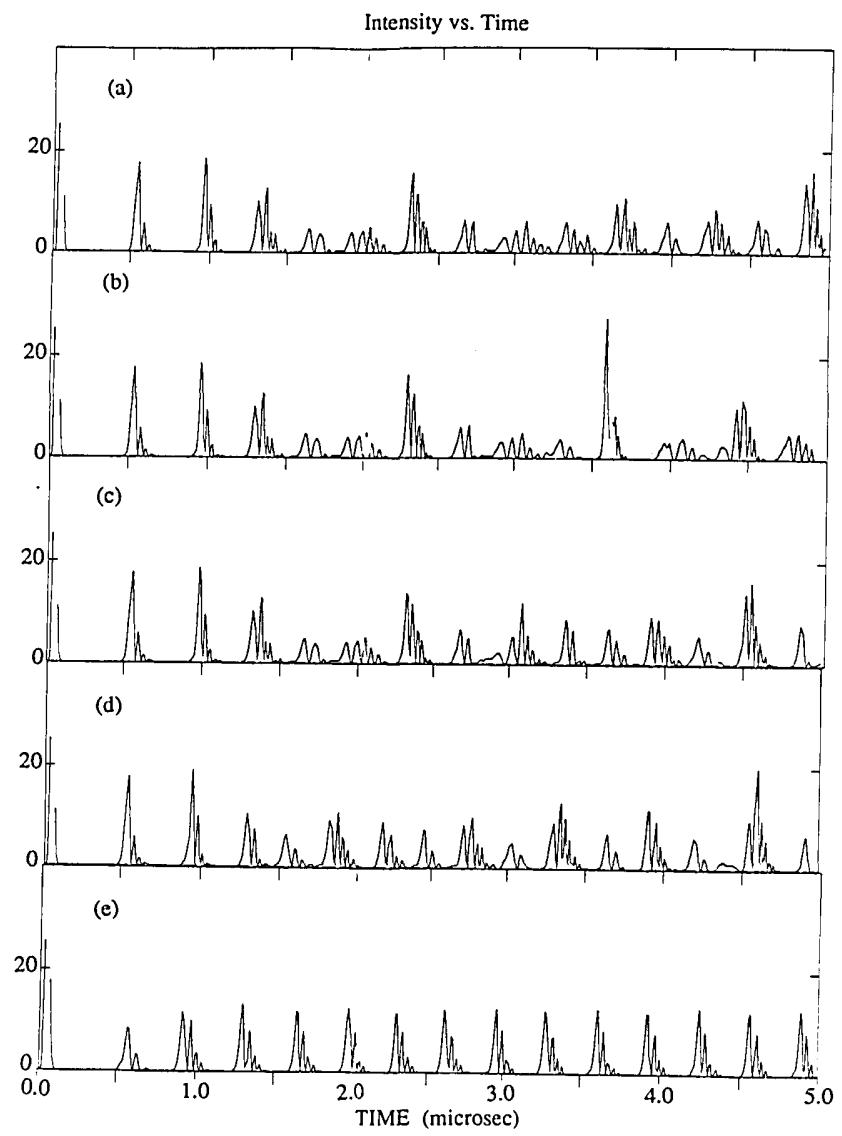

Fig. 3. Theoretical spontaneous pulsation intensity waveforms for a standing-wave laser with threshold parameter $r=2.0$, line-center tuning $\left(y_{0}=0\right)$, and number of spatial harmonics corresponding to (a) $k_{\max }=8$, (b) $k_{\max }=6$, (c) $k_{\max }=4$, (d) $k_{\max }=2$, (e) $k_{\max }=1$. The time period for which accurate predictions can be made becomes shorter for smaller values of $k_{\max }$.

ties in some of the parameters and initial conditions for any experiment that one might wish to model.

The waveforms in Figs. 3(a)-3(d) are actually quite similar qualitatively, and for $r=2.0$ it is probably never necessary to use a $k$ value larger than $k_{\max }=2$. This is also true for other values of $r$, especially those that correspond to chaotic pulsations. The sensitive dependence on the initial conditions of such behavior would result in slightly different irregular pulsations for a slight change of initial conditions. With $k_{\max }=1$ the pulsations are apparently more regular than for larger numbers of spatial harmonics. However, even this apparent discrepancy may be deceptive, since pulsation burst regularity is also sensitively dependent on the value of the threshold parameter. In many practical situations the initial conditions and other parameters would not be known well enough to permit a meaningful distinction between pulsation waveforms like those shown in Figs. 3(d) and 3(e).

As one would expect, fewer spatial harmonics are required when a laser is operated closer to threshold. Figure 4 shows theoretical plots of the intensity for a xenon laser that is tuned to line center and operated at a threshold parameter value of $r=1.1$. It is evident that the pulse shapes are much simpler for operation close to threshold. The waveforms are now indistinguishable for $k_{\max }>4$ [Fig. 4(c)], and errors with $k_{\max }=2$ [Fig. 4(d)] are barely perceptible at $5 \mu \mathrm{sec}$ ( 6 bursts). For typical applications even the $k_{\max }=1$ [Fig. 4(e)] approximation would seem to be entirely adequate. Truncated spatial harmonic expansions have also been used in rate-equation modeling of laser dynamics. ${ }^{10,11}$

In the limit $k_{\max }=1$ the equations governing the standing-wave laser are substantially simplified. In particular, Eqs. (1)-(6) reduce to

$$
\begin{aligned}
\frac{\partial P_{r, 1}(V, t)}{\partial t}= & -\gamma\left[(1+i V) P_{r, 1}(V, t)+y P_{i, 1}(V, t)\right. \\
& \left.+i A_{i}(t) D_{0}(V, t)\right], \\
\frac{\partial P_{i, 1}(V, t)}{\partial t}= & -\gamma\left[(1+i V) P_{i, 1}(V, t)-y P_{r, 1}(V, t)\right. \\
& \left.-i A_{r}(t) D_{0}(V, t)\right] \\
\frac{\partial D_{0}(V, t)}{\partial t}= & \lambda_{a}(V)-\lambda_{b}(V)-h_{1} D_{0}(V, t)-h_{2} M_{0}(V, t) \\
& -2 \gamma_{1}\left[A_{r}(t) P_{i, 1 i}(V, t)-A_{i}(t) P_{r, 1 i}(V, t)\right] \\
& +\frac{\varepsilon \Gamma_{a}}{2 \pi^{1 / 2}} \exp \left(-\varepsilon^{2} V^{2}\right) \\
& \times \int_{-\infty}^{\infty}\left[M_{0}\left(V^{\prime}, t\right)+D_{0}\left(V^{\prime}, t\right)\right] \mathrm{d} V^{\prime} \\
& -\frac{\varepsilon \Gamma_{b}}{2 \pi^{1 / 2}} \exp \left(-\varepsilon^{2} V^{2}\right) \\
& \times \int_{-\infty}^{\infty}\left[M_{0}\left(V^{\prime}, t\right)-D_{0}\left(V^{\prime}, t\right)\right] \mathrm{d} V^{\prime},
\end{aligned}
$$

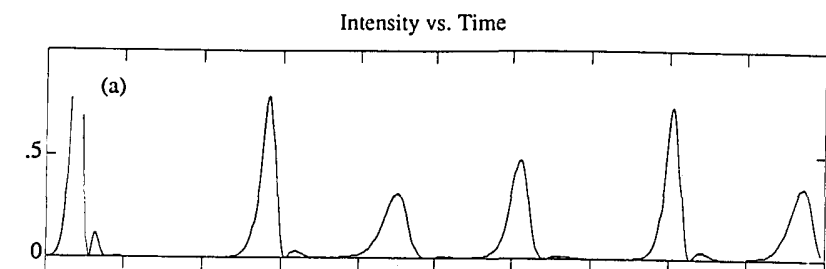

(b)
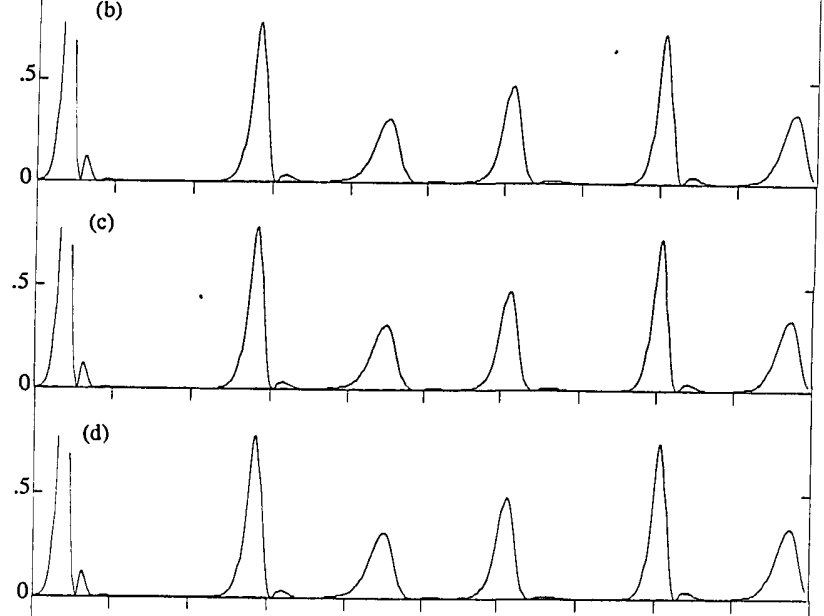

(e)

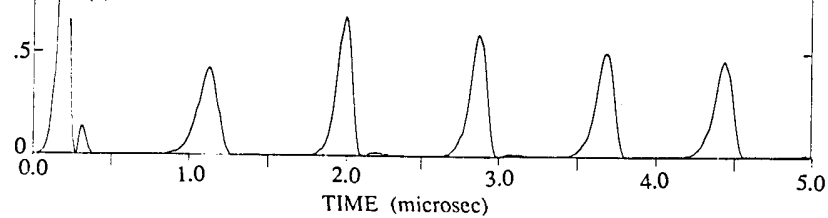

Fig. 4. Theoretical intensity waveforms for a laser with $r=1.1$ and $y_{0}=0$ and the number of spatial harmonics corresponding to (a) $k_{\max }=8$, (b) $k_{\max }=6$, (c) $k_{\max }=4$, (d) $k_{\max }=2$, (e) $k_{\max }=1$. As the threshold parameter is reduced, fewer spatial harmonics are required for accurate results. 
Intensity vs. Time

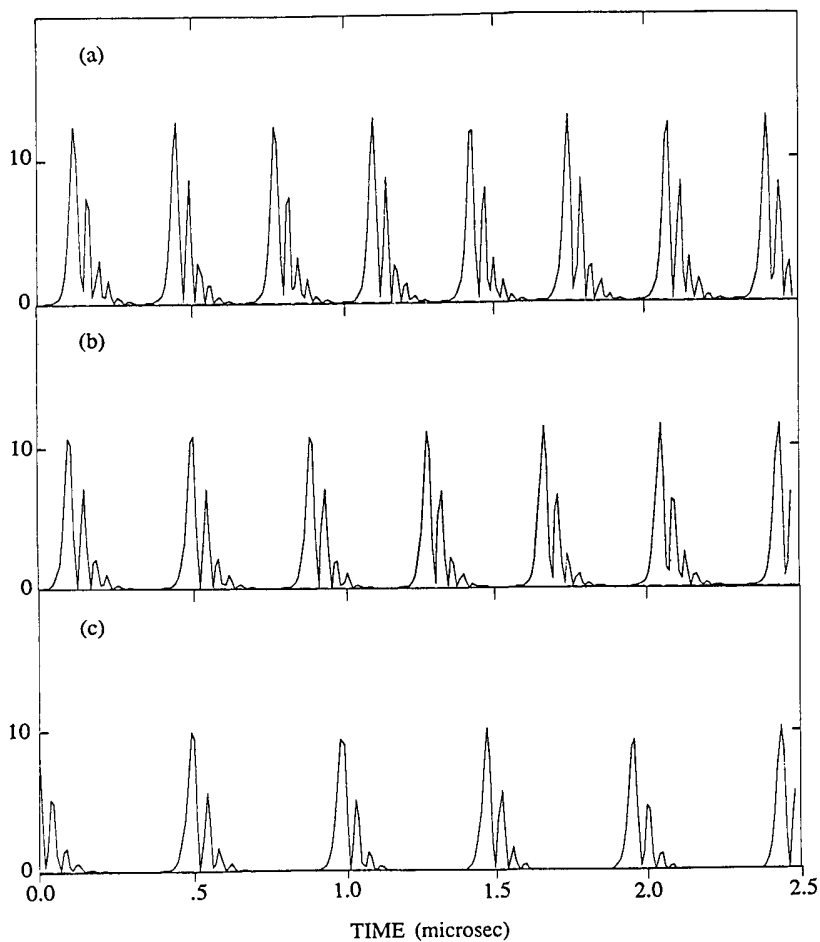

Intensity vs. time

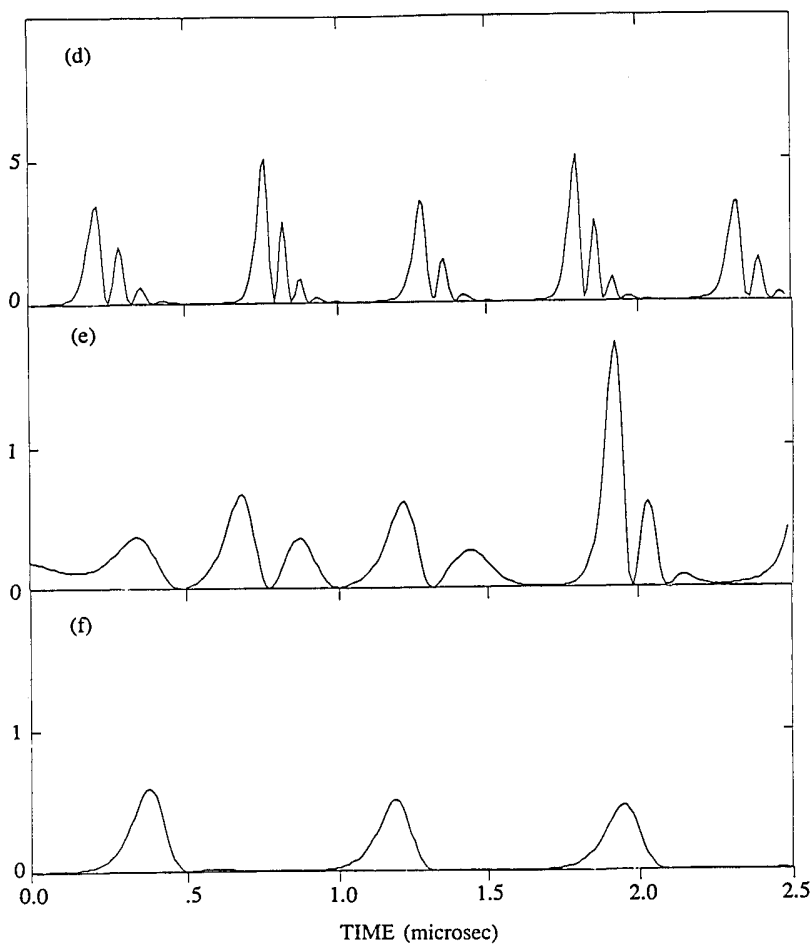

Fig. 5. Theoretical intensity waveforms for a laser with line-center tuning, $k_{\max }=1$, and threshold parameter values (a) $r=2.0$, (b) $r=$ 1.8 , (c) $r=1.6$, (d) $r=1.4$, (e) $r=1.2$, (f) $r=1.1$. Generally the pulsation amplitude, frequency, and waveform complexity decrease with decreasing values of $r$.

$$
\begin{aligned}
\frac{\partial M_{0}(V, t)}{\partial t}= & \lambda_{a}(V)+\lambda_{b}(V)-h_{3} M_{0}(V, t)-h_{4} D_{0}(V, t) \\
& +\frac{\varepsilon \Gamma_{a}}{2 \pi^{1 / 2}} \exp \left(-\varepsilon^{2} V^{2}\right) \\
& \times \int_{-\infty}^{\infty}\left[M_{0}\left(V^{\prime}, t\right)+D_{0}\left(V^{\prime}, t\right)\right] \mathrm{d} V^{\prime} \\
& +\frac{\varepsilon \Gamma_{b}}{2 \pi^{1 / 2}} \exp \left(-\varepsilon^{2} V^{2}\right) \\
& \times \int_{-\infty}^{\infty}\left[M_{0}\left(V^{\prime}, t\right)-D_{0}\left(V^{\prime}, t\right)\right] \mathrm{d} V^{\prime} \\
\frac{\mathrm{d} A_{r}(t)}{\mathrm{d} t}= & -\frac{1}{2 t_{c}}\left[A_{r}(t)+\delta\left(y-y_{0}\right) A_{i}(t)\right. \\
& \left.-\int_{-\infty}^{\infty} P_{i, 1 i}(V, t) \mathrm{d} V\right] \\
& \left.+\int_{-\infty}^{\infty} P_{r, 1 i}(V, t) \mathrm{d} V\right] \\
\mathrm{d} A_{i}(t) & \frac{1}{2 t_{c}}\left[A_{i}(t)-\delta\left(y-y_{0}\right) A_{r}(t)\right. \\
& \\
&
\end{aligned}
$$

where for analysis it is more convenient to use the original equations rather than the computationally more efficient set given as Eqs. (26)-(33). Also, the pump rates are again assumed to be constant. While these equations look similar to those that preceded them, the absence here of the $k$ (or $j$ ) subscript means that we are now dealing with a smaller number of equations.
While the neglect of the higher spatial harmonics leads to a dramatic simplification of the standing-wave model, it is important to note that even this simplifed model is substantially different from the corresponding ring laser model as given by Eqs. (1)-(6) of Ref. 8. The polarization elements in the above standing-wave model are complex, and only in special cases do the standing-wave and ring models become essentially equivalent. One such case, involving line-center tuning, is noted in Section 6 below.

\section{OTHER SIMPLIFICATIONS}

The theoretical model discussed above contains many complications besides multiple spatial harmonics. While some of these complications may be important when one seeks quantitative agreement between theory and experiment, they may not all be required for more qualitative investigations. The purpose of this section is to explore the effects of several additional simplifications of the theoretical model in much the same way as was done in an investigation of a semiclassical model for ring laser instabilities. $^{8}$ The plots in Fig. 5 give an overview of the types of pulsation that can be observed in a xenon laser model with $k_{\max }=1$ for various values of the threshold parameter. This set of plots will serve as a reference point for the more approximate results to be described in the following paragraphs.

One important complication of the model is the inclusion of spectral cross relaxation arising from velocitychanging collisions. When the cross-relaxation terms in Eqs. (64)-(69) are switched off, one is left with simpler equations for $D_{0}(V, t)$ and $M_{0}(V, t)$. The resulting set of equations is 


$$
\begin{aligned}
& \frac{\partial P_{r, 1}(V, t)}{\partial t}=-\gamma\left[(1+i V) P_{r, 1}(V, t)+y P_{i, 1}(V, t)\right. \\
& \left.+i A_{i}(t) D_{0}(V, t)\right] \\
& \frac{\partial P_{i, 1}(V, t)}{\partial t}=-\gamma\left[(1+i V) P_{i, 1}(V, t)-y P_{r, 1}(V, t)\right. \\
& \text { - } \left.i A_{r}(t) D_{0}(V, t)\right] \text {, } \\
& \frac{\partial D_{0}(V, t)}{\partial t}=\lambda_{a}(V)-\lambda_{b}(V)-\frac{\gamma_{a}+\gamma_{a b}+\gamma_{b}}{2} D_{0}(V, t) \\
& -\frac{\gamma_{a}+\gamma_{a b}-\gamma_{b}}{2} M_{0}(V, t)-\frac{4 \gamma_{a} \gamma_{b}}{\gamma_{a}-\gamma_{a b}+\gamma_{b}} \\
& \times\left[A_{r}(t) P_{i, 1 i}(V, t)-A_{i}(t) P_{r, 1 i}(V, t)\right] \text {, } \\
& \frac{\partial M_{0}(V, t)}{\partial t}=\lambda_{a}(V)+\lambda_{b}(V)-\frac{\gamma_{a}-\gamma_{a b}+\gamma_{b}}{2} M_{0}(V, t) \\
& -\frac{\gamma_{a}-\gamma_{a b}-\gamma_{b}}{2} D_{0}(V, t) \\
& \frac{\mathrm{d} A_{r}(t)}{\mathrm{d} t}=-\frac{1}{2 t_{c}}\left[A_{r}(t)+\delta\left(y-y_{0}\right) A_{i}(t)\right. \\
& \left.-\int_{-\infty}^{\infty} P_{i, 1 i}(V, t) \mathrm{d} V\right], \\
& \frac{\mathrm{d} A_{i}(t)}{\mathrm{d} t}=-\frac{1}{2 t_{c}}\left[A_{i}(t)-\delta\left(y-y_{0}\right) A_{r}(t)\right. \\
& \left.+\int_{-\infty}^{\infty} P_{r, 1 i}(V, t) \mathrm{d} V\right]
\end{aligned}
$$

where Eqs. (7)-(11) were used to eliminate the $h$ parameters and $\gamma_{1}$.

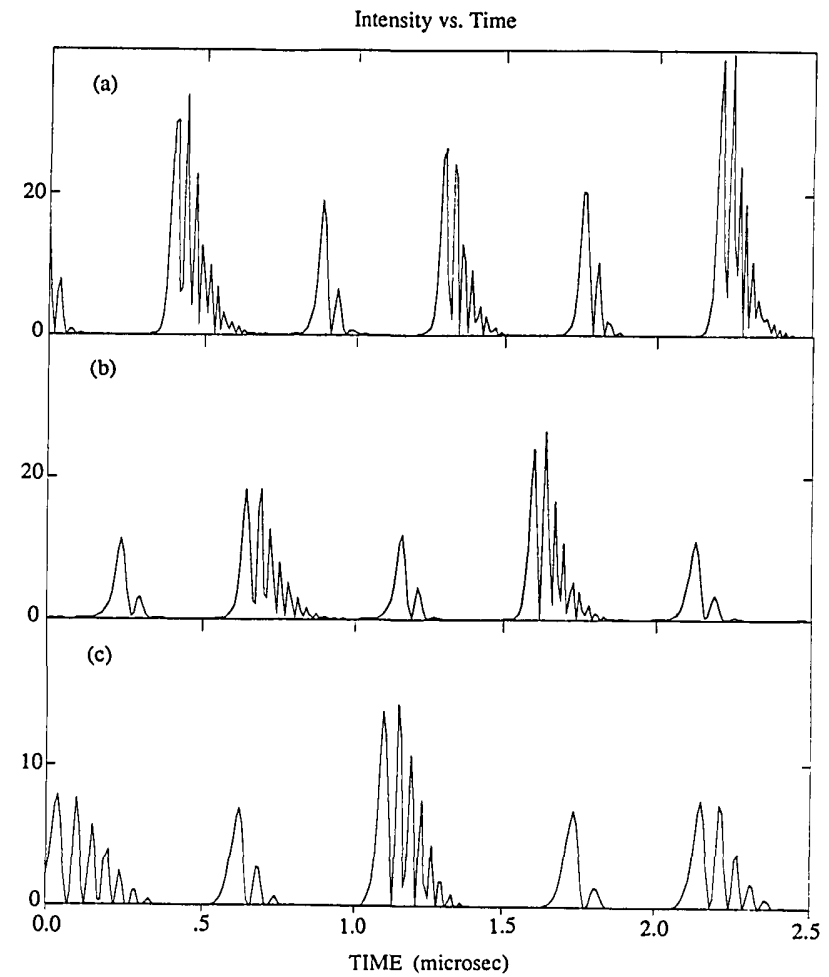

A set of pulsation waveforms based on Eqs. (70)-(75) with line-center tuning is shown in Fig. 6. These pulsations should be compared with the pulsations shown in Fig. 5. As in the ring laser case, there are significant effects of cross relaxation, including the speeding up and stabilization of the pulsations. Even though the neglect of spectral cross relaxation reduces the quantitative accuracy of the model, most qualitative features of the instability are retained.

Another complication of the analysis concerns the energy-level model, which permits arbitrary total decay rates from the upper and lower laser levels as well as an arbitrary rate between levels. One finds that the essence of the laser instability can still be retained with simpler energy-level schemes. For example, if $\gamma_{b}$ is set equal to $\gamma_{a}+\gamma_{a b}$ then Eq. (72) becomes independent of the parameter $M_{0}(V, t)$. In this case the equation for $M_{0}(V, t)$ can be ignored, and Eqs. (70)-(75) reduce to

$$
\begin{aligned}
\frac{\partial P_{r, 1}(V, t)}{\partial t}=-\gamma\left[(1+i V) P_{r, 1}(V, t)+y P_{i, 1}(V, t)\right. & \\
& \left.+i A_{i}(t) D_{0}(V, t)\right], \\
\frac{\partial P_{i, 1}(V, t)}{\partial t}=-\gamma\left[(1+i V) P_{i, 1}(V, t)-y P_{r, 1}(V, t)\right. & \\
& \left.\quad-i A_{r}(t) D_{0}(V, t)\right], \\
\frac{\partial D_{0}(V, t)}{\partial t}= & \lambda_{a}(V)-\lambda_{b}(V)-\left(\gamma_{a}+\gamma_{a b}\right) D_{0}(V, t) \\
& -2\left(\gamma_{a}+\gamma_{a b}\right)\left[A_{r}(t) P_{i, 1 i}(V, t)-A_{i}(t) P_{r, 1 i}(V, t)\right],
\end{aligned}
$$

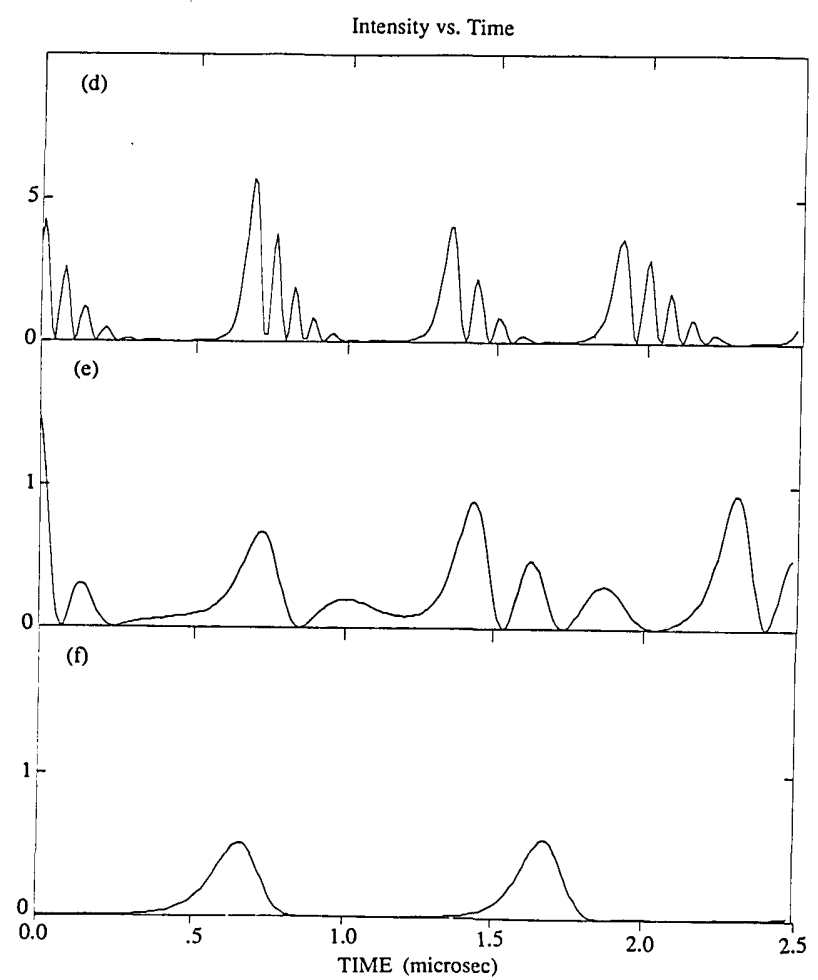

Fig. 6. Theoretical intensity waveforms for a laser with line-center tuning and $h_{\max }=1$ but with spectral cross relaxation neglected. The threshold parameter values are (a) $r=2.0$, (b) $r=1.8$, (c) $r=1.6$, (d) $r=1.4$, (e) $r=1.2$, and (f) $r=1.1$. Comparison with data such as those shown in Fig. 5 indicates that spectral cross relaxation causes a modest increase in pulsation frequency but no major qualitative
change in the general pulsation characteristics. 


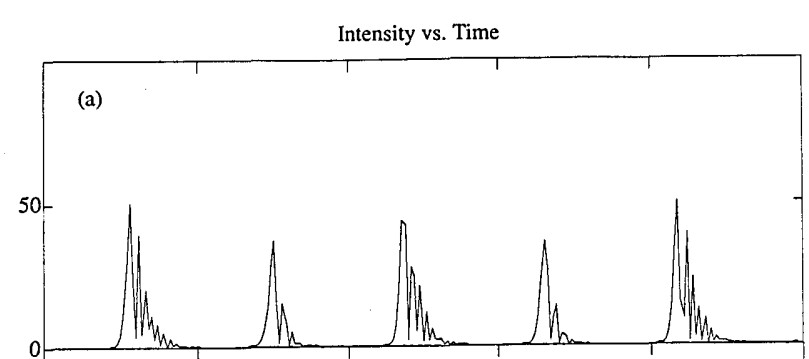

(b)

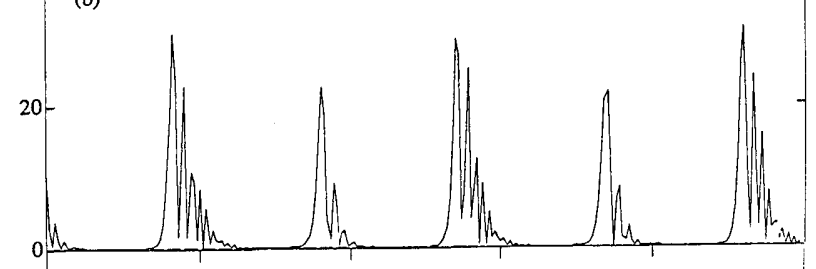

(c)

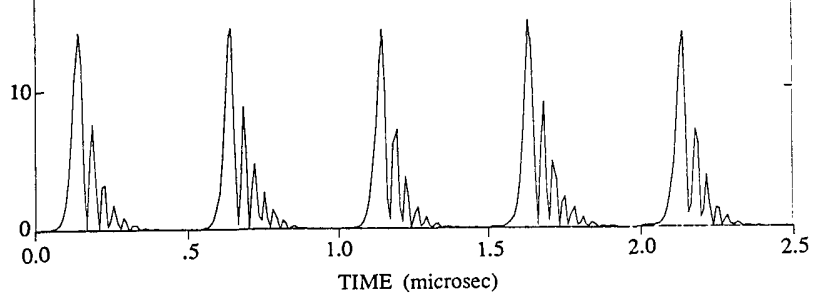

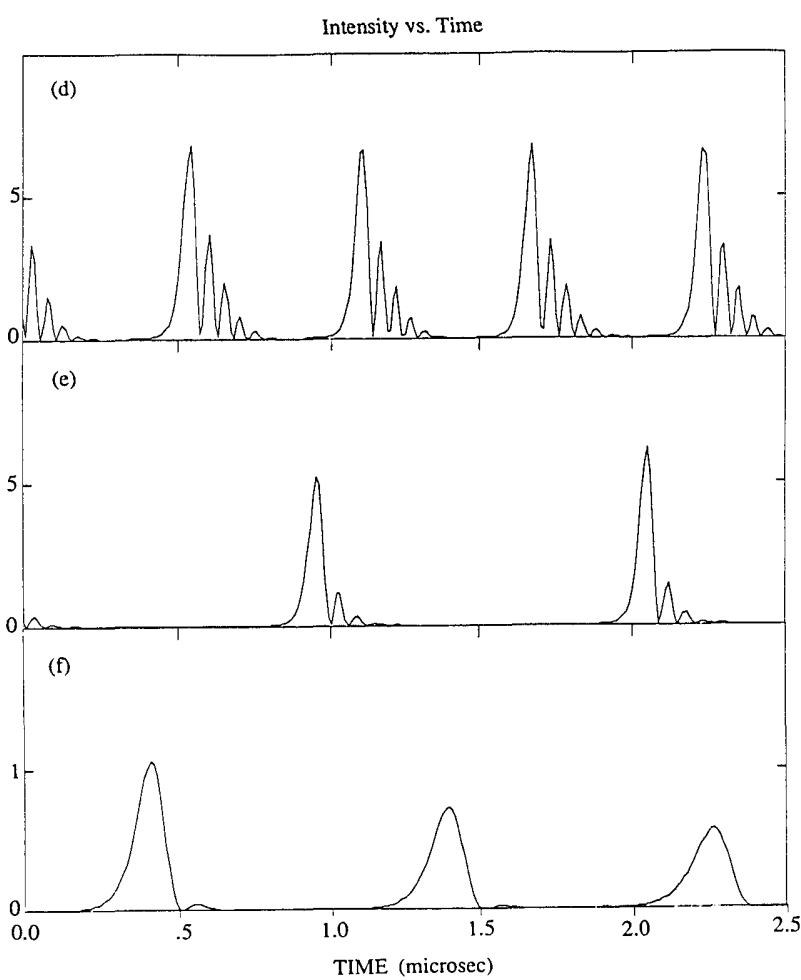

Fig. 7. Theoretical intensity waveforms for a laser with line-center tuning but with simplified energy-level structure and with spectral cross relaxation neglected. The threshold parameter values are (a) $r=2.0$, (b) $r=1.8,(\mathrm{c}) r=1.6$, (d) $r=1.4$, (e) $r=1.2$, (f) $r=1.1$.

$$
\begin{aligned}
\frac{\mathrm{d} A_{r}(t)}{\mathrm{d} t}= & -\frac{1}{2 t_{c}}\left[A_{r}(t)+\delta\left(y-y_{0}\right) A_{i}(t)\right. \\
& \left.-\int_{-\infty}^{\infty} P_{1,1 i}(V, t) \mathrm{d} V\right], \\
\frac{\mathrm{d} A_{i}(t)}{\mathrm{d} t}= & -\frac{1}{2 t_{c}}\left[A_{i}(t)-\delta\left(y-y_{0}\right) A_{r}(t)\right. \\
& \left.+\int_{-\infty}^{\infty} P_{r, 1 i}(V, t) \mathrm{d} V\right] .
\end{aligned}
$$

Figure 7 includes a series of pulsation waveforms based on Eqs. (76)-(80) with line-center tuning for various values of the threshold parameter. These waveforms should be compared with those of Fig. 6, which include the more realistic energy decay rates. One finds from this comparison that simplifying the decay rates has little qualitative effect, and hence for many purposes the simpler model may be adequate.

In all the numerical results that have been described, it can be observed that the intensity and other system parameters never vary significantly within the cavity decay time $t_{c}=1.0 \mathrm{nsec}$. This suggests that setting the time derivatives to zero in the field equations [Eqs. (79)(80)] may be an appropriate approximation. If this is done, the fields can be written explicitly in terms of the polarizations according to

$$
\begin{aligned}
& A_{r}(t)=\frac{\delta\left(y-y_{0}\right) \int_{-\infty}^{\infty} P_{r, 1 i}(V, t) \mathrm{d} V+\int_{-\infty}^{\infty} P_{i, 1 i}(V, t) \mathrm{d} V}{1+\delta^{2}\left(y-y_{0}\right)^{2}}, \\
& A_{i}(t)=\frac{\delta\left(y-y_{0}\right) \int_{-\infty}^{\infty} P_{i, 1 i}(V, t) \mathrm{d} V-\int_{-\infty}^{\infty} P_{r, 1 i}(V, t) \mathrm{d} V}{1+\delta^{2}\left(y-y_{0}\right)^{2}} .
\end{aligned}
$$

With these substitutions the electric fields can be eliminated from Eqs. (76)-(78).

It is of value also to consider the dramatic simplifications of the model that occur with line-center tuning $\left(y=y_{0}=0\right)$. At line center Eqs. (81) and (82) reduce to

$$
\begin{aligned}
& A_{r}(t)=+\int_{-\infty}^{\infty} P_{i, 1 i}(V, t) \mathrm{d} V, \\
& A_{i}(t)=-\int_{-\infty}^{\infty} P_{r, 1 i}(V, t) \mathrm{d} V .
\end{aligned}
$$

When these results are inserted into Eqs. (76)-(78), one obtains

$$
\begin{aligned}
\frac{\partial P_{r, 1}(V, t)}{\partial t}=-\gamma\left[(1+i V) P_{r, 1}(V, t)\right. & \\
& \left.-i D_{0}(V, t) \int_{-\infty}^{\infty} P_{r, 1 i}(V, t) \mathrm{d} V\right], \\
\frac{\partial P_{i, 1}(V, t)}{\partial t}=-\gamma\left[(1+i V) P_{i, 1}(V, t)\right. & \left.-i D_{0}(V, t) \int_{-\infty}^{\infty} P_{i, 1 i}(V, t) \mathrm{d} V\right], \\
\frac{\partial D_{0}(V, t)}{\partial t}= & \lambda_{d}(V)-\gamma_{d} D_{0}(V, t) \\
& -2 \gamma_{d}\left[P_{i, 1 i}(V, t) \int_{-\infty}^{\infty} P_{i, 1 i}(V, t) \mathrm{d} V\right. \\
& \left.+P_{r, 1 i}(V, t) \int_{-\infty}^{\infty} P_{r, 1 i}(V, t) \mathrm{d} V\right],
\end{aligned}
$$

where for compactness the pump and population difference decay rates are denoted $\lambda_{d}$ and $\gamma_{d}$, respectively. From 
the symmetry of these equations it is clear that one can obtain nontrivial solutions while arbitrarily setting the phase of the complex polarization amplitude. For example, with $P_{r, 1}=0$, Eqs. (85)-(87) can be replaced by the real set

$$
\begin{aligned}
& \frac{\partial P_{i, 1 r}(V, t)}{\partial t}=-\gamma\left[P_{i, 1 r}(V, t)-V P_{i, 1 i}(V, t)\right], \\
& \frac{\partial P_{i, 1 i}(V, t)}{\partial t}=-\gamma\left[P_{i, 1 i}(V, t)+V P_{i, 1 r}(V, t)\right. \\
& \left.-D_{0}(V, t) \int_{-\infty}^{\infty} P_{i, 1 i}(V, t) \mathrm{d} V\right], \\
& \frac{\partial D_{0}(V, t)}{\partial t}=\lambda_{d}(V)-\gamma_{d} D_{0}(V, t) \\
& -2 \gamma_{d}\left[P_{i, 1 i}(V, t) \int_{-\infty}^{\infty} P_{i, 1 i}(V, t) \mathrm{d} V\right] \text {. }
\end{aligned}
$$

It may be noted that this set of equations is almost the model, which was given as Eqs. (33)-(35) of Ref. 8. The only difference is the presence of the factor 2 in Eq. (90), and this 2 shows that, at the present level of approximation, saturation of the population difference at line center occurs twice as fast in a standing-wave laser as it does in a unidirectional ring laser. This faster saturation occurs because in a standing-wave laser the atoms interact with both the right- and left-traveling waves.

The simplification at line center that was just discussed could actually have been incorporated much earlier if one were interested only in line-center operation. Thus with $y=y_{0}=0$ it is possible to set $P_{r, 2 j+1}(V, t)=0$ and

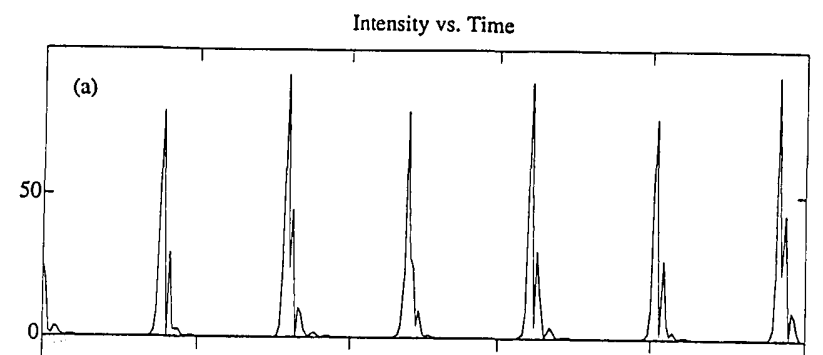

(b)

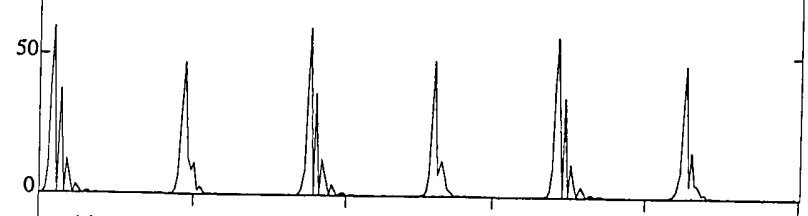

(c)

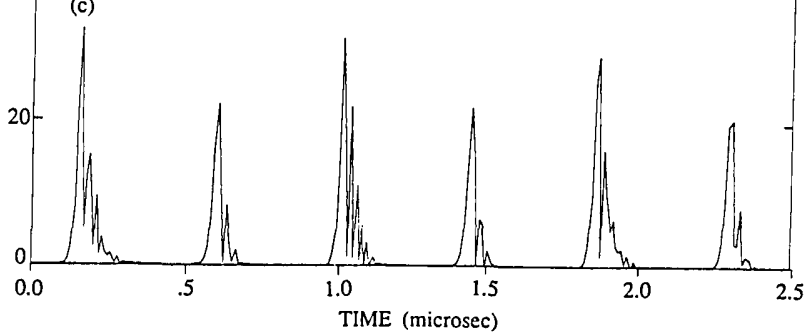
same as the corresponding set for a simplified ring laser

$A_{i}(t)=0$ in Eqs. (1)-(6). However, for the more quantitative applications of the model one would not want to be restricted to line-center operation, and thus this limitation was withheld until the last step.

A set of waveform solutions of Eqs. (88)-(90) is shown in Fig. 8, in which the same parameter values as those in Fig. 7 are used. Except for minor quantitative differences, the neglect of the electric-field derivatives has no major effect on the pulsation waveforms. More generally, none of the approximations discussed above alters the basic instability behavior of the lasers. Consequently, for some applications the simplest models should prove to be adequate, and the same conclusion was reached in a study of unidirectional ring lasers. ${ }^{8}$

\section{RATE-EQUATION APPROXIMATION}

The laser models considered in Sections 6 and 7 all reduced the complexity of the laser description without substantially altering the fundamental instability characteristics. For completeness we also briefly examine the rate-equation approximation, in which the polarization is assumed to be an instantaneous function of the electric field. The most general version of this approximation can be obtained by setting to infinity the polarization decay rate $\gamma$ in Eqs. (1) and (2) while requiring the derivatives to remain finite. In this way Eqs. (1) and (2) can be solved explicitly for the polarization components, to obtain

$$
\begin{array}{r}
P_{r, 2 j+1}(V, t)=-i \frac{\left\{[1+(2 j+1) i V] A_{i}(t)+y A_{r}(t)\right\}}{[1+(2 j+1) i V]^{2}+y^{2}} \\
\times\left[D_{2 j}(V, t)-D_{2 j+2}(V, t)\right],
\end{array}
$$

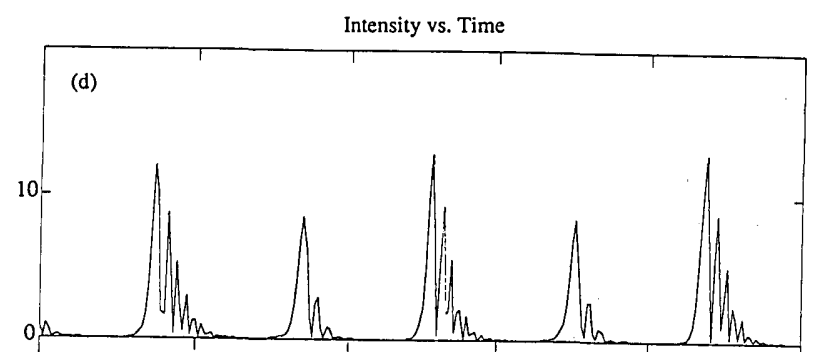

(e)

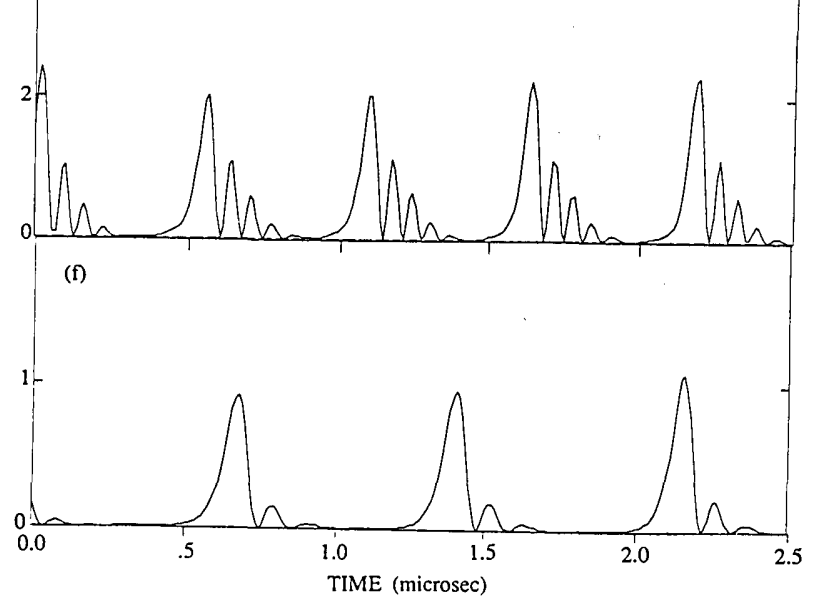

Fig. 8. Theoretical intensity waveforms for a laser with line-center tuning but with field derivatives and spectral cross relaxation neglected and with a simplified energy-level structure. The threshold parameter values are (a) $r=2.0$, (b) $r=1.8$, (c) $r=1.6$, (d) $r=1.4$, (e) $r=1.2$, (f) $r=1.1$. Comparison with Fig. 7 indicates that the neglect of the field derivatives in this example has little effect on the 

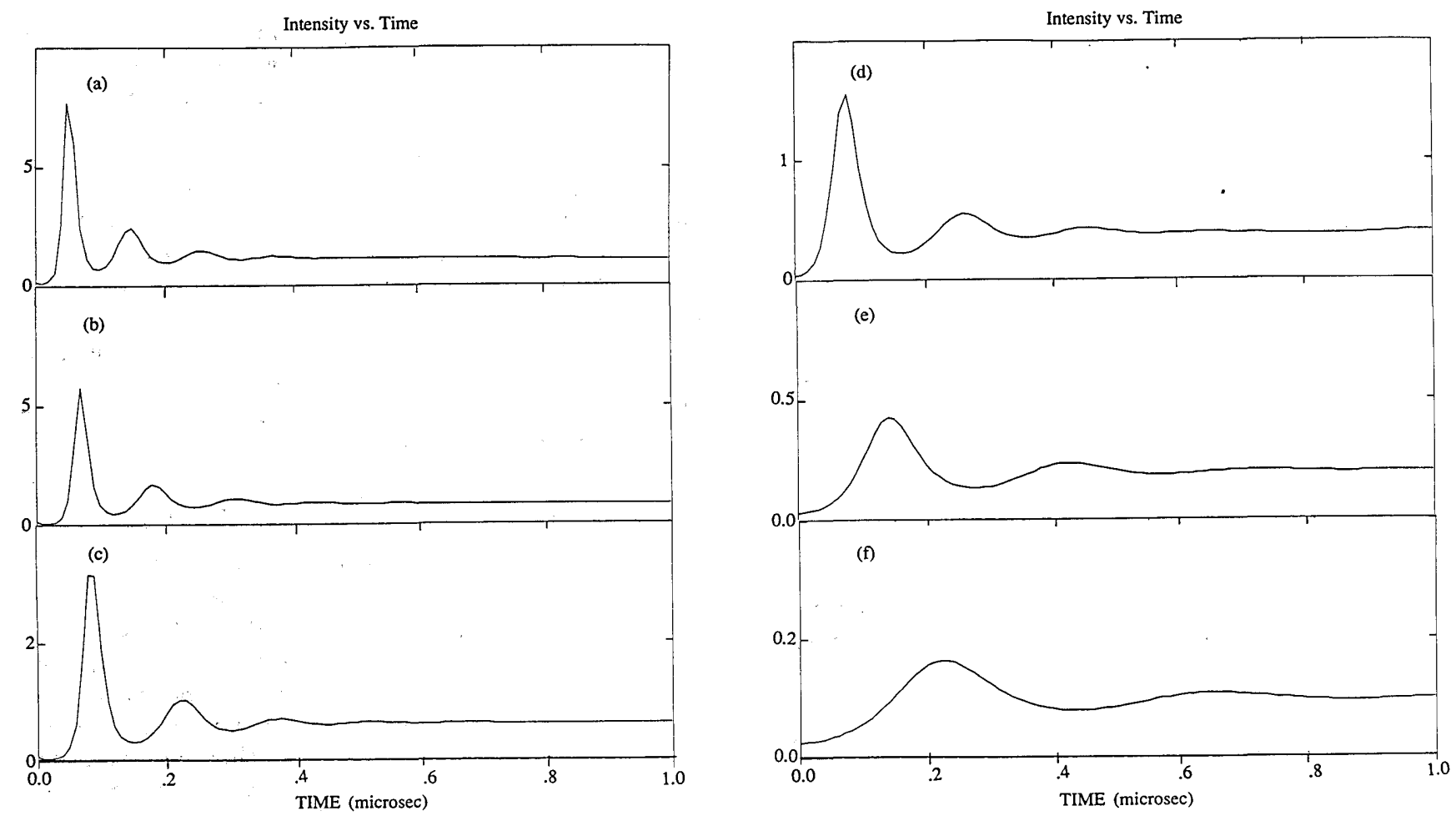

Fig. 9. Theoretical intensity waveforms for a laser with line-center tuning in the rate-equation approximation. The threshold parameter values are (a) $r=2.0$, (b) $r=1.8$, (c) $r=1.6$, (d) $r=1.4$, (e) $r=1.2$, (f) $r=1.1$. In the rate-equation approximation the relaxation oscillations are always damped.

$$
\begin{array}{r}
\left.P_{i, 2 j+1}(V, t)=-i \frac{\left\{-[1+(2 j+1) i V] A_{r}(t)+y A_{i}(t)\right\}}{[1+}(2 j+1) i V\right]^{2}+y^{2} \\
\times\left[D_{2 j}(V, t)-D_{2 j+2}(V, t)\right] .
\end{array}
$$

When these results are substituted into Eqs. (3)-(6), the results are rate equations for a standing-wave laser.

The rate equations that have just been described may be solved numerically, and a series of solutions for the xenon laser is shown in Fig. 9. The laser is always stable, and the resulting damped relaxation oscillations are in contrast to the pulsations exhibited by all our semiclassical models. None of the more complicated energy-level structure, the spectral cross relaxation, the inhomogeneous broadening, or the spatial variations of the standing-wave fields acts to destabilize the rate equation model. The rate-equation approximation should never be used if there is any possibility of semiclassical instabilities.

\section{CONCLUSION}

The semiclassical instabilities that are sometimes observed in inhomogeneously broadened lasers are becoming increasingly well understood, and in the case of the 3.51$\mu \mathrm{m}$-wavelength xenon laser quantitative agreement between theory and experiment seems to be possible. In this study we have started from a standing-wave model and explored the consequences of several approximations, including reduced longitudinal spatial hole burning, simplified energy-level structure, and neglect of spectral cross relaxation and electric-field derivatives. These approximations may lead to substantial savings in computa- tion time, and they may sometimes be justifiable on physical grounds or in terms of their observed minimal effects. On the other hand, the rate-equation approximation should never be used if there is any reason to anticipate the occurrence of a semiclassical instability.

\section{ACKNOWLEDGMENTS}

This research was supported in part by the National Science Foundation and by Tektronix, Inc.

*Present address, National Institute of Physics, University of the Philippines, Diliman, Quezon City, Philippines.

\section{REFERENCES}

1. L. W. Casperson, IEEE J. Quantum Electron. QE-14, 756 (1978).

2. L. W. Casperson, in Third New Zealand Symposium on Laser Physics, J. D. Harvey and D. F. Walls, eds., Vol. 182 of Springer Lecture Notes in Physics (Springer-Verlag, Berlin, 1983), p. 88.

3. N. B. Abraham, L. A. Lugiato, and L. M. Narducci, J. Opt. Soc. Am. B 2, 7 (1985).

4. R. W. Boyd, M. G. Raymer, and L. M. Narducci, eds., Optical Instabilities (Cambridge U. Press, Cambridge, 1986).

5. N. B. Abraham, F. T. Arecchi, and L. A. Lugiato, eds., Instabilities and Chaos in Quantum Optics II (Plenum, New York, 1988).

6. L. W. Casperson, J. Opt. Soc. Am. B 5, 958 (1988).

7. L. W. Casperson, J. Opt. Soc. Am. B 5, 970 (1988).

8. L. W. Casperson, J. Opt. Soc. Am. B 2, 73 (1985).

9. L. W. Casperson, Phys. Rev. 21, 911 (1980).

10. Y. I. Khanin, in Ref. 4, p. 212.

11. M. G. Raymer, Z. Deng, and M. Beck, J. Opt. Soc. Am. B 5, 1588 (1988). 